\title{
Deletion of meso-2,3-butanediol dehydrogenase gene budC for enhanced $D$-2,3-butanediol production in Bacillus licheniformis
}

\author{
Gaofu Qi ${ }^{1}$, Yanfang Kang ${ }^{1}$, Lu Li', Aifang Xiao', Shumeng Zhang ${ }^{1}$, Zhiyou Wen ${ }^{2,3}$, Dihong Xu
} and Shouwen Chen ${ }^{1 *}$

\begin{abstract}
Background: D-2,3-butanediol has many industrial applications such as chiral reagents, solvents, anti-freeze agents, and low freezing point fuels. Traditional D-2,3-butanediol producing microorganisms, such as Klebsiella pneumonia and K. xoytoca, are pathogenic and not capable of producing D-2,3-butanediol at high optical purity. Bacillus licheniformis is a potential 2,3-butanediol producer but the wild type strain (WX-02) produces a mix of $D$ - and meso-type isomers. BudC in B. licheniformis is annotated as 2,3-butanediol dehydrogenase or acetoin reductase, but no pervious experiment was performed to verify this hypothesis.

Results: We developed a genetically modified strain of B. licheniformis (WX-02 $\Delta$ budC) as a D-2,3-butanediol producer with high optimal purity. A marker-less gene deletion protocol based on a temperature sensitive knock-out plasmid T2-Ori was used to knock out the budC gene in B. licheniformis WX-02. The budC knock-out strain successfully abolished meso-2,3-butanediol production with enhanced D-2,3-butanediol production. No meso-BDH activity was detectable in cells of this strain. On the other hand, the complementary strain restored the characteristics of wild strain, and produced meso-2,3-butanediol and possessed meso-BDH activity. All of these data suggested that budC encoded the major meso-BDH catalyzing the reversible reaction from acetoin to meso-2,3-butanediol in $B$. licheniformis. The budC knock-out strain produced D-2,3-butanediol isomer only with a high yield of $30.76 \mathrm{~g} / \mathrm{L}$ and a productivity of $1.28 \mathrm{~g} / \mathrm{L}-\mathrm{h}$.
\end{abstract}

Conclusions: We confirmed the hypothesis that budC gene is responsible to reversibly transfer acetoin to meso-2,3-butanediol in B. licheniformis. A mutant strain of B. licheniformis with depleted budC gene was successfully developed and produced high level of the D-2,3-butanediol with high optimal purity.

Keywords: Bacillus licheniformis, D-2,3-butanediol, BudC gene, meso-2,3-butanediol dehydrogenase

\section{Background}

D-2,3-butanediol as one of the promising bulk chemicals has extensive applications in cosmetics, foods, transport fuels, medicines, and polymers industries [1]. In general, 2,3-butanediol exists in three stereoisomeric forms: $D-2$, 3-butanediol, L-2,3-butanediol and meso-2,3-butanediol [2]. All these isomers are valuable chemicals that provide chiral groups in drugs [3]. D-2,3-butanediol is also used as an antifreeze agent because of its low freezing point

\footnotetext{
* Correspondence: mel212@126.com

${ }^{1}$ State Key Laboratory of Agricultural Microbiology, College of Life Science and Technology, Huazhong Agricultural University, Wuhan 430070, China Full list of author information is available at the end of the article
}

$\left(-60^{\circ} \mathrm{C}\right)[1]$. The production of 2,3-butanediol with high optical purities is therefore highly desirable $[4,5]$.

Although many microorganisms are capable of synthesizing 2,3-butanediol, the production processes are hindered by various limitations. For example, traditional 2,3-butanediol producing microorganisms, such as Klebsiella pneumonia and $K$. xoytoca, are pathogenic $[2,6]$ and produce a mixture of meso- and $L$-isomers with low yield and productivity [2,7]. Non-pathogenic species such as Paenibacillus polymyxa can produce D-2,3-butanediol with a high (up to 98\%) enantioselective purity; however, the cell density and the overall $D$-2,3-butanediol productivity is low as the cells need to be grown in micro-aerobic 
conditions [1,3]. The growth of $P$. polymyxa also needs yeast extract and tryptone, which increases the medium cost and the production recovery cost [8]. Bacillus licheniformis, which is a generally-regarded-as-safe (GRAS) organism, is also capable of producing 2,3-butanediol at the industrial level $[6,9,10]$; however, the wild-type $B$. licheniformis produces a mix of $D$ - and meso-2,3-butanediol isomers [6].

The metabolic pathway from pyruvate to 2,3-butanediol has been well studied in B. subtilis. As shown in Figure 1, pyruvate is converted to $\alpha$-acetolactate, and consequently to acetoin. At high dissolved-oxygen and glucose-rich conditions, acetoin can be further converted into 2,3-butanediol by the enzyme called acetoin reductase (AR). The same protein can catalyze the reverse reaction from 2 , 3-butanediol to acetoin as well, when dissolved oxygen is limited and glucose is depleted. In this case, however, the enzyme is called 2,3-butanediol dehydrogenase (BDH) $[1,11]$. The meso-AR/BDH is encoded by the $b d h \mathrm{~A}$ gene in $B$. subtilis, therefore, modification of the bdhA gene may be an efficient way to increase the optical purity of $D$-2,3-butanediol while avoiding the formation of meso2,3-butanediol by B. subtilis [1].

Research has been attempted to produce 2,3-butanediol with high optical purity using genetically engineered microorganisms. For example, Nielsen et al. [12] introduced the acetoin and meso-2,3-butanediol biosynthesis pathway in Escherichium coli by co-expression of 2,3butanediol dehydrogenase originally derived from yeast, resulting in $1.12 \mathrm{~g} / \mathrm{L}$ meso-2,3-butanediol [12]. Li et al. [13] also transferred the gene encoding 2,3-butanediol dehydrogenase from Enterobacter cloacae to E. coli for expressing $L$-2,3-butanediol from diacetyl with concentrations of $16.1 \mathrm{~g} / \mathrm{L}$ and $26.8 \mathrm{~g} / \mathrm{L}$ of $L$-2,3-butanediol produced in batch and fed-batch fermentation, respectively. Although production of high optical purity of 2 , 3-butanediol isomers has been achieved in engineered E. coli, the product yield was usually very low, mainly due to the weak overflow of the metabolic pathway in E. coli cells.

Compared to E. coli, Bacillus species such as B. licheniformis, B. subtilis, and B. amyloliquefaciens have a strong overflow metabolic pathway from glucose. Therefore, modification of the metabolic pathway of Bacillus species provides a promising way for producing pure 2,3-butanediol isomer with high product titer. In our previous work, we have isolated a strain of $B$. licheniformis (termed WX-02), which showed a rapid growth and capability of producing $\gamma$-poly-glutamic acid $(\gamma$-PGA) accompanied with 2,3-butanediol and acetoin [14]. Similar to $B$. subtilis and other B. licheniformis strains, however, B. licheniformis WX-02 produces a mixture of D-2,3-butanediol and meso-2,3-butanediol [11,14,15]. Furthermore, the genome of $B$. licheniformis WX-02 was sequenced and the data submitted [GenBank: AHIF01000000], but a gene similar to the $b d h$ A gene in $B$. subtilis was not found in $B$. licheniformis WX-02. The gene budC (gene ID: 3100198) in B. licheniformis WX-02 genome is annotated as AR;

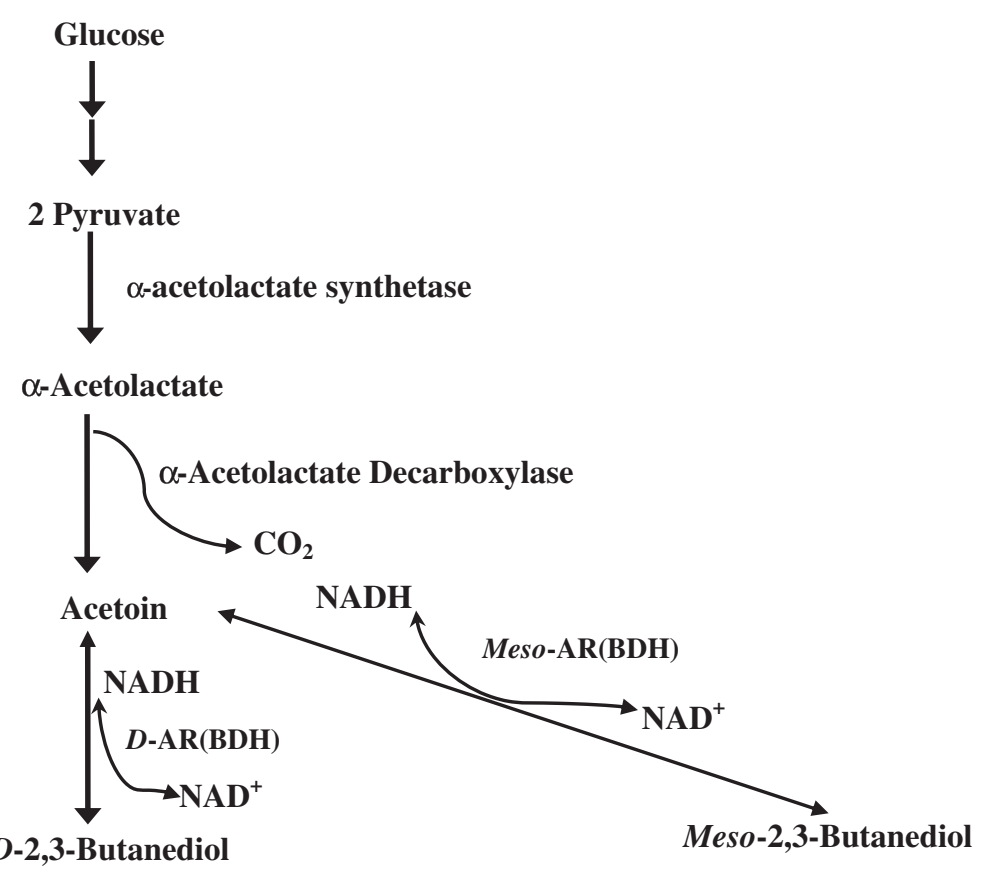

Figure 1 Metabolic pathways to acetoin and 2,3-butanediol optical isomers in B. licheniformis. AR, acetoin reductase (forward reaction); $\mathrm{BDH}, 2,3$-butanediol dehydrogenase (reverse reaction); $\mathrm{NADH}$, nicotinamide adenine dinucleotide. 
this gene $(\operatorname{budC})$ is the same as that of $B$. licheniformis ATCC 14580 (DSM 13) [16], although it has little similarity (1.67\% identity aligned by UniProt (http://www. uniprot.org/?tab=align)) to bdhA in B. subtilis. The cell extract of $B$. licheniformis also shows AR (BDH) activity, with acetoin, $D$-2,3-butanediol and meso-2,3-butanediol also being identified. All these results indicate the existence of the gene encoding $\mathrm{AR}(\mathrm{BDH})$ in B. licheniformis $[17,18]$. Recent research by Li et al. [10] also shows that the recombinant $E$. coli containing the $\mathrm{BDH}$ and glycerol dehydrogenase (GDH) encoding gene from $B$. licheniformis exhibited meso-BDH and D-BDH activity in vitro [10]. The objective of this work was to investigate the specific function of budC in the metabolism of acetoin and 2,3butanediol in B. licheniformis WX-02, followed with developing a strategy of knocking out the budC gene so the production of the sole $D$-2,3-butanediol isomer can be achieved.

\section{Results}

\section{Establishment of the budC gene knock-out strain and} complementary strain

Figure 2 shows the plasmids for deletion of the budC gene and for construction of the complementary strain. These two plasmids were used for transformation of B. licheniformis WX-02 for making the budC deletion mutant and its complementary strain, respectively. The positive strains were then verified by PCR and sequencing.

The PCR results for verification of budC knock-out are shown in Figure 3A. The PCR product amplified from the genomic DNA of budC knock-out strain was about 1,500 bp; while a DNA fragment of about 2200 bp containing the budC gene and its up- and down- stream sequences was amplified from the genomic DNA of wild strain WX-02 by using primers of $\Delta b u d C$-F and $\Delta b u d C-\mathrm{R}$ as negative control. The PCR product from budC knockout strain was purified and sequenced, and no other mutation than the budC deletion was found (data not shown), suggesting the successful construction of the budC deficient strain (WX-02 $\Delta b u d C$ ). The complementary strain of WX-02 $\Delta b u d C$ (terms as WX-02 $\Delta$ budC/pHYbudC), was also verified by PCR. As shown in Figure 3B, a DNA fragment of about 1500 bp was amplified from the recombinant plasmid of transformant with a matched size to the fusion fragment of P43-budC-TamyL, and then further verified by DNA sequencing (data not shown).

\section{Effect of budC knock-out on meso-AR/meso and BDH activities}

As described in Figure 1, the enzyme catalyzing the conversion between acetoin and 2,3-butanediol exhibits two activities depending on the culture conditions: AR activity for reduction of acetoin to 2,3-butanediol and BDH activity for dehydrogenation of 2,3-butanediol to acetoin. To investigate the effect of budC knock-out on AR and $\mathrm{BDH}$ activities, strains WX-02, WX-02 $\Delta b u d \mathrm{C}$ and WX$02 \Delta b u d \mathrm{C} / \mathrm{pHY} b u d \mathrm{C}$ were cultured for 12,24 and $36 \mathrm{~h}$; the specific activities of meso-BDH, AR, and D-BDH in the cell extracts were analyzed. As shown in Figure 4A, no meso-BDH activity was detected in the cell extracts of WX-02 $\triangle b u d C$ throughout the culture; whereas both WX-02 and WX-02 $\Delta b u d C / p H Y b u d C$ exhibited a high meso-BDH activity. As for the AR activity, WX-02 $\Delta b u d \mathrm{C}$ exhibited a very weak AR activity as compared to the other two strains (WX-02 and WX-02 $\Delta b u d \mathrm{C} / \mathrm{pHY}$ HudC) (Figure 4B). Figure $4 \mathrm{C}$ shows that $D-\mathrm{BDH}$ activity of WX$02 \Delta b u d C$ was comparable to that of WX-02 and WX-02 $\Delta b u d \mathrm{C} / \mathrm{pHY}$ budC (Figure 4C). Collectively, the above results indicate that the deletion of the budC gene had a
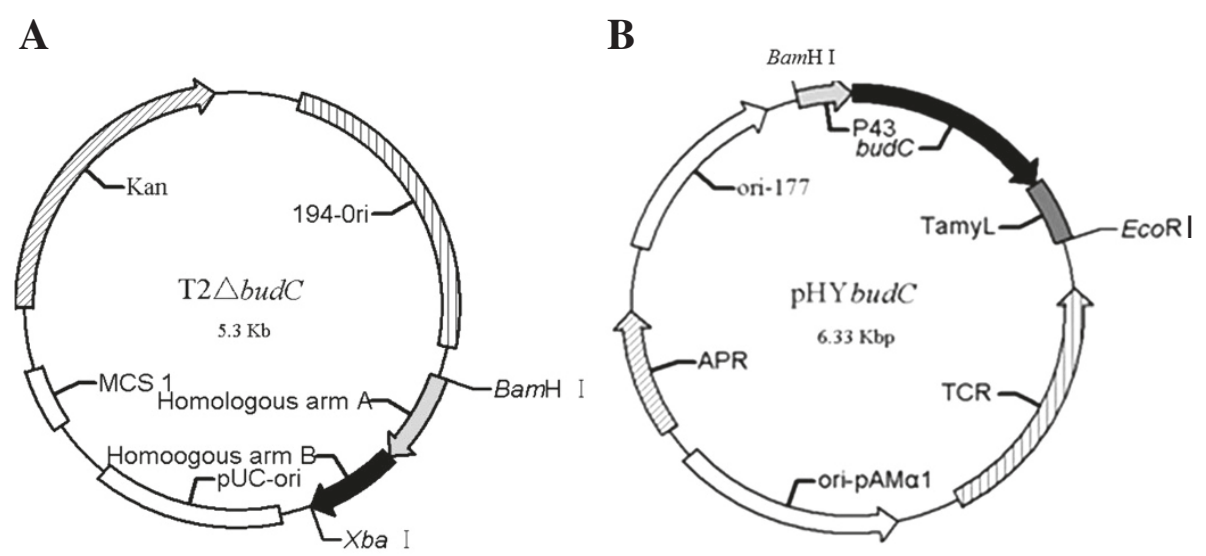

Figure 2 Construction of recombinant vectors. (A) Recombinant vector T2 $\Delta$ budC for budC knock-out. The vector contained a temperaturesensitive replicon from B. subtilis (194-Ori), Kanamycin-resistant gene (Kan), and the homologous arm A and B for homologous recombination. (B) Recombinant vector of pHYbudC for expression of budC in B. licheniformis. The vector contained budC expression cassette including P43 promoter, the budC gene and the a-amylase gene (amyL) transcription terminator of B. licheniformis WX-02. 

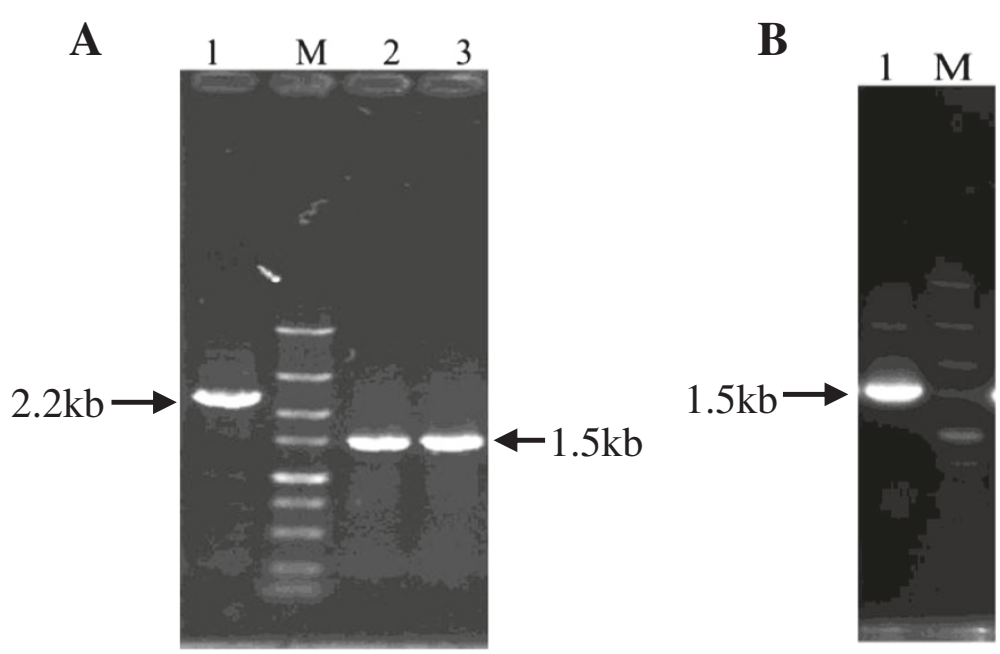

Figure 3 PCR verification of recombinant strains. (A) PCR verification of budC knock-out strain. M, DL5000 marker $(5,000,3,000,2,000$, 1,500, 1,000, 750, 500, 250 and $100 \mathrm{bp}$, up to bottom); lane 1, negative control (PCR product from the genomic DNA of B. licheniformis WX-02); lanes 2 and 3, fragment amplified from the genomic DNA of B. licheniformis WX-02 $\Delta$ budC. (B) PCR verification of complementary strain of $B$. licheniformis WX-02 $\Delta$ budC/pHYbudC. M, DL5000 marker; lane 1, PCR product of fusion fragment of P43-budC-TamyL from recombinant strain B. licheniformis WX-02 $\Delta$ budC/pHYbudC.

significant effect on meso-BDH, but not on $D$-BDH activity, indicating that the budC gene encodes meso-BDH but not $D-\mathrm{BDH}$. It should also be noted that WX-02 $\Delta b u d \mathrm{C} /$ pHYbudC restored both meso-AR and meso-BDH activities compared to the budC gene knock-out strain; these two enzyme activities in WX-02 $\Delta b u d \mathrm{C} / \mathrm{pHY} b u d \mathrm{C}$ were higher than those in WX-02 (Figure 4A and B). The reason may be due to the multicopy of the $b u d C$ gene controlled by a strong promoter of P43 in WX-02 $\Delta b u d C /$ pHYbudC strain as compared to the wild-type strain [19].

Effects of budC deletion on 2,3-butanediol configurations Among the three stereoisomers of 2,3-butanediol, $D$ - and $L$-types are racemic and can only be separated in a chiral column; they can be easily separated from the meso-type by ordinary non-chiral gas chromatograph (GC) capillary columns [4]. In this study, therefore, $D$-2,3-butanediol and meso-2,3-butanediol produced by B. licheniformis $[2,17]$ were separated by ordinary non-chiral GC.

Strains WX-02, WX-02 $\Delta b u d C$ and WX-02 $\Delta b u d C /$ $\mathrm{pHYbudC}$ were respectively cultured for $24 \mathrm{~h}$ and then 2,3-butanediol in broth was determined. As shown in Figure 5, D-2,3-butanediol and meso-2,3-butanediol were well-separated. WX-02 $\Delta b u d C$ produced $D$-2,3-butanediol but no meso-2,3-butanediol, whereas WX-02 and WX-02 $\Delta b u d C /$ pHYbudC generated both $D$-2,3-butanediol and meso-2,3-butanediol (Figure 5). The result clearly shows that the synthesis of meso-2,3-butanediol in budC knocked-out strain (WX-02 $\Delta b u d C$ ) was successfully deleted, whereas the complementation of the budC knock-out strain (WX-02 $\Delta b u d \mathrm{C} / \mathrm{pHY}$ budC) restored the capability of meso-2,3-butanediol synthesis. The result confirmed that the budC gene was responsible for meso-2,3-butanediol production in $B$. licheniformis. Unexpectedly, the transformed strain (WX-02 $\Delta b u d \mathrm{C} / \mathrm{pHY} b u d \mathrm{C}$ ) had a higher meso-BDH activity, but this strain did not produce more meso-2,3-butanediol than the wild-type (WX-02). We believe that the synthesis of meso-2,3-butanediol in this transformant may be controlled by other rate-limiting factors. For example, the conversion from acetoin to meso-2,3butanediol also needs nicotinamide adenine dinucleotide $(\mathrm{NADH})$ as the electro-donor [6,18]; this $\mathrm{NADH}$ in the transformant may be the controlling factor, although the activity of meso-BDH is higher in the strain.

\section{Production of 2,3-butanediol and acetoin by budC knock-out strain and wild-type strain}

The production profile of 2,3-butanediol and acetoin by the wild strain and budC knock-out strain are presented in Figure 6. As shown in Figure 6A, wild-strain WX-02 produced both $D$ - and meso-types of 2,3-butanediol throughout the culture. The concentration of these two isomers increased in the first $24 \mathrm{~h}$; beyond this culture time, $D-2,3-$ butanediol concentration decreased whereas meso-2,3butanediol leveled off in the remainder of the culture period. For the culture of WX-02 $\Delta b u d C$ strain, however, only the $D$-type of 2,3-butanediol was produced throughout all the culture. The $D$-2,3-butanediol concentration increased for the first $24 \mathrm{~h}$, and decreased afterwards. Only a trace amount of meso-2,3-butanediol was detected at the end of culture ( $48 \mathrm{~h}$ ) of WX-02 $\Delta b u d \mathrm{C}$. Throughout all the culture period, the concentration of $D$-2,3-butanediol in the WX-02 $\Delta b u d C$ culture was approximately the summation of the $D$ - and meso-2,3-butanediol isomers in the 

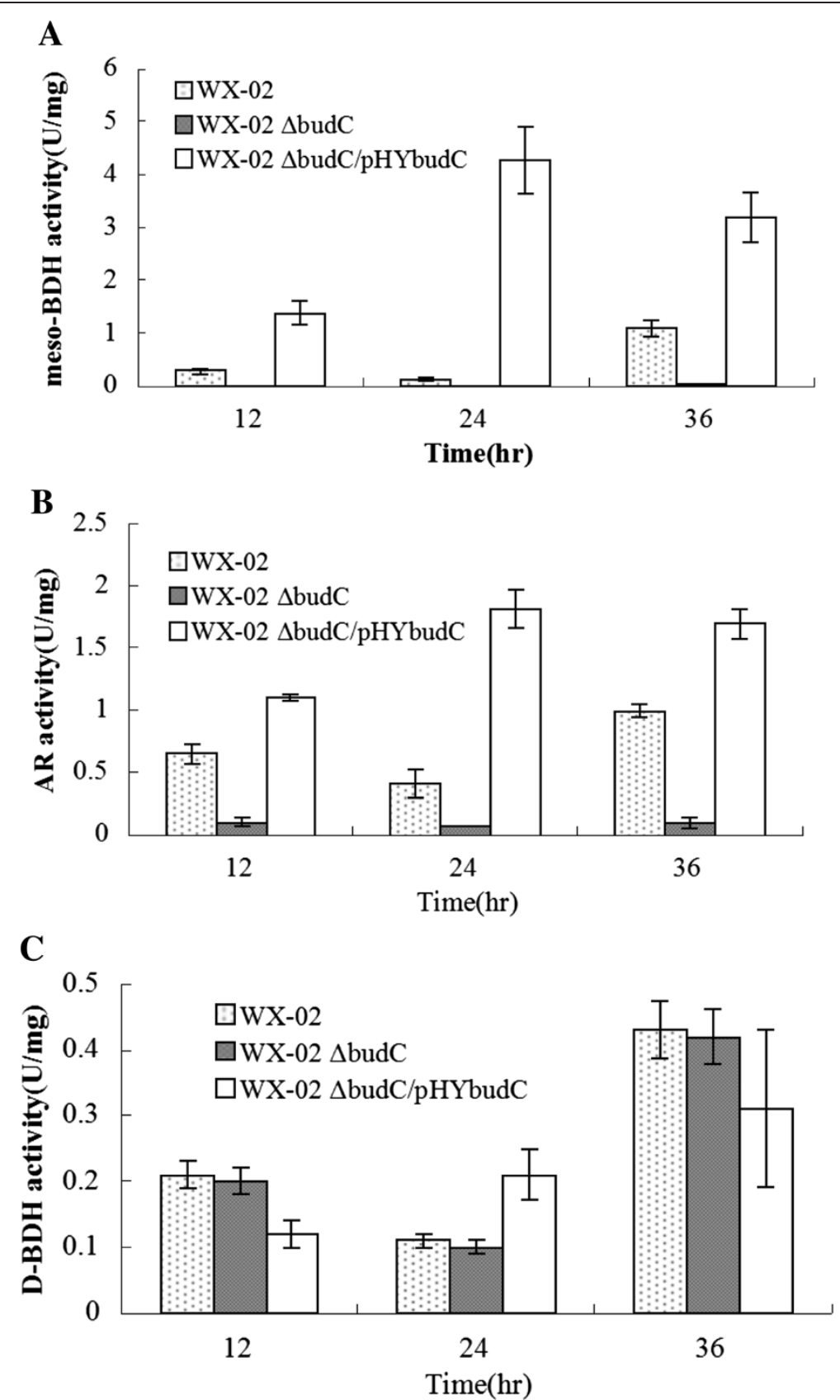

Figure 4 Activity of 2,3-butanediol dehydrogenas (BDH) and acetoin reductase (AR) in different $B$. licheniformis. (A) meso-BDH activity; (B) AR activity; (C) D-BDH activity. The data are expressed as mean \pm standard error of three replicates.

WX-02 culture, indicating the meso-2,3-butanediol originally formed in the wild-type cells switched to additional $D$-2,3-butanediol in the WX-02 $\Delta b u d C$ mutant. The above production profiles for $D$ - and meso-type of 2,3-butanediol were also reported in the culture of B. subtilis and Serratia marcescens $[9,20]$.

As for the production of acetoin and total 2,3-butanediol, Figure 6B shows that for both the wild-type and budC gene knock-out strain, total 2,3-butanediol production increased rapidly in the first $24 \mathrm{~h}$ and gradually decreased afterwards; concurrently, the acetoin production of the two strains was low in the first $24 \mathrm{~h}$, but increased rapidly from 24 to $36 \mathrm{~h}$. The loss of the budC gene in the WX-02 $\Delta b u d C$ strain resulted in more acetoin accumulation than wild strain after $36 \mathrm{~h}$.

Figure $6 \mathrm{C}$ shows $\mathrm{pH}$ change, glucose utilization, and biomass density of the two strain cultures. The $\mathrm{pH}$ values were low in the first $24 \mathrm{~h}$, indicating the synthesis of 


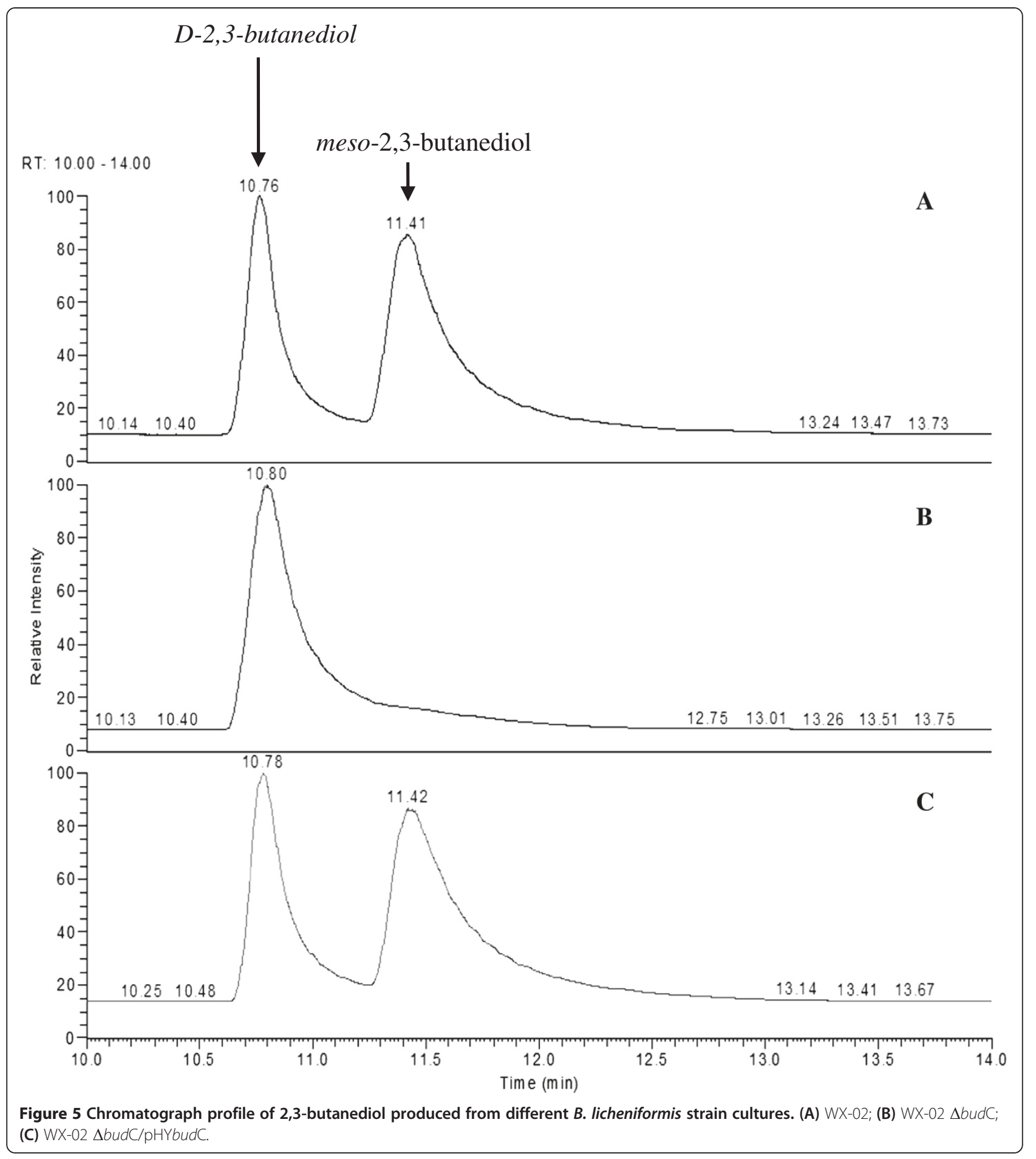

organic acids by the strains. This low $\mathrm{pH}$ level favored the synthesis of 2,3-butanediol (Figure 6B). In the later stage of culture, the slight increase in $\mathrm{pH}$ favored the conversion of $D$-2,3-butanediol to acetoin, which is evidenced by the increased concentration of acetoin in the medium (Figure 6B). The similar trend between $\mathrm{pH}$ and acetoin/ 2,3-butanediol conversion was also found in B. subtilis
[21]. Figure $6 \mathrm{~B}$ also shows that glucose for the two cultures had a similar trend; the glucose was rapidly consumed within the first $24 \mathrm{~h}$, which corresponds to a rapid cell growth in the two cultures. After $24 \mathrm{~h}$ the glucose in the medium was almost depleted; as a result the cells of both the wild-type strain and budC-gene knockout mutant ceased growth due to the glucose (Figure $6 \mathrm{C}$ ). 

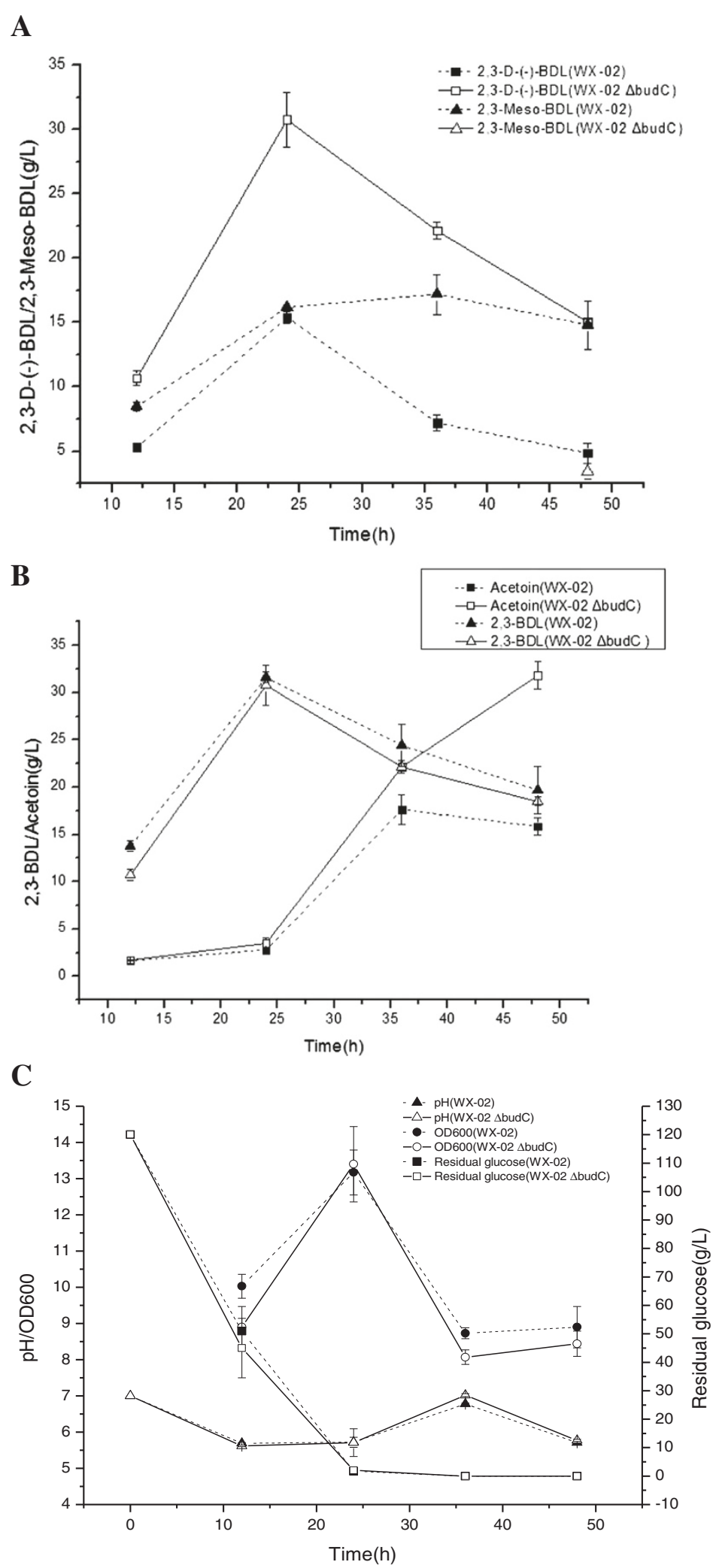

Figure 6 Comparison of metabolites production, cell growth, and glucose consumption profile by wild-type (WX-02) and budC knock-out (WX-02 $\Delta$ budC) B. licheniformis strains. (A) D- and meso-2,3-butanediol production. (B) Total 2,3-butanediol and acetoin production. (C) Cell density, medium $\mathrm{pH}$, and residual glucose concentration. Data are expressed as mean \pm standard errors of three replicates. BDL, butanediol. 
However, the wild-type cells showed a higher cell density than the mutant; the reason was probably due to the consumption of acetoin by the wild-type strain for supporting the cell growth. Indeed, it has been reported that acetoin can be a good carbon source for the culture of $B$. licheniformis once the major carbon source, glucose, is depleted $[20,22]$. This may also explain why the acetoin concentration in the mutant culture was much higher than that in the wild-type strain (Figure 6B).

\section{Discussion}

As a valuable compound, $D$-2,3-butanediol has been widely used as a major composition in solvents, antifreeze agents, synthetic rubber, and plastics [2]. It can also be used as a potential fuel with a low freezing point and its heating value is comparable to that of ethanol and methanol [6]. Various efforts have been attempted for producing optical purity of $D$-2,3-butanediol by genetically modified microorganisms; however, the yield of $D$-2,3-butanediol has still been very low. For example, recombinant $E$. coli expressing the enzyme BDH was found to produce $6.1 \mathrm{~g} / \mathrm{L}$ of $D$-2,3-butanediol [21], and $B$. licheniformis with a deleted lactate dehydrogenase gene $(l d h)$ to produce $13.77 \mathrm{~g} / \mathrm{L}$ of $D$-2,3-butanediol [6]. Heterologous expression of acetoin reductase of Clostridium beijerinckii in C. acetobutylicum has resulted in a range of 1.8 to $1.98 \mathrm{~g} / \mathrm{L} D$-2,3-butanediol [7].

The strain B. licheniformis, WX-02, used in this study was previously isolated for the production of $\gamma$-PGA with 2,3-butanediol and acetoin being the co-products. This strain can grow in a simple medium containing glucose, glutamic acid, and mineral salts [14]. As the strain WX-02 produces mixed stereoisomers of 2,3-butanediol, modification of its metabolic pathway for sole production of pure isomer of $D$-2,3-butanediol is desirable. To date, there have been several challenges to making a recombinant strain of $B$. licheniformis, including low transformation efficiency and a lack of information about the meso-BDH encoding gene. For example, Wang et al. [6] successfully deleted the $l d h$ gene from genomic DNA in $B$. licheniformis by transforming protoplasts of the cells with a recombinant knock-out plasmid; however, the designed protoplast system was very complicated and the transformation efficiency was low [6]. In this paper, we successfully transformed $B$. licheniformis WX-02 with a recombinant knock-out plasmid with high efficiency. It demonstrates that T2-ori-based knock-out plasmid and the electro-transformation approach can be used for the metabolic modification of the $B$. licheniformis WX-02 strain for producing pure $D$ 2,3-butanediol with high titer.

Previous reports showed that the $b d h \mathrm{~A}$ gene encoding $\mathrm{BDH}$ is responsible for catalyzing acetoin to 2,3-butanediol in B. subtilis, and the insertion inactivation of $b d h \mathrm{~A}$ completely blocks 2,3-butanediol synthesis [11]. For $B$. licheniformis, however, no bdhA gene was found in the genome. In the attempt for identifying the gene responsible for catalyzing acetoin to 2,3-butanediol in B. licheniformis, Wang et al. [6] reported the depletion of the $l d h$ gene for the production of high optical purity of $D$ 2,3-butanediol in B. licheniformis with $13.77 \mathrm{~g} / \mathrm{L} D-2,3-$ butanediol being produced in optimized conditions [6]. However, our previous study in knocking out the $l d h$ gene in B. licheniformis WX-02 to block lactate accumulation resulted in reduced acetoin and 2,3-butanediol production (unpublished data). The recent report showed that the budC gene might be the gene encoding meso$\mathrm{BDH}$ in B. licheniformis according to the detectable enzyme activity of the recombinant protein of $b d h$ (same as budC) gene by E. coli [10]. Therefore, we hypothesized that the $b u d C$ gene is responsible for catalyzing acetoin to meso-2,3-butanediol in B. licheniformis.

Our previous research shows that the budC gene in $B$. licheniformis might be annotated as $\mathrm{BDH}$ for this species [16]. In this work, we confirmed the budC in B. licheniformis as the gene encoding meso-BDH for the reversible reaction from acetoin to meso-2,3-butanediol [18], based on the fact that the deletion of budC gene in B. licheniformis WX-02 completely blocked meso-2,3-butanediol production with significant enhanced production of $D$ 2,3-butanediol (Figure 6A). However, the BudC protein sequence [NCBI: YP_006713433.1], aligned by blastP in the NCBI non-redundant protein database, showed that only one protein from $B$. sonorensis annotated as 2,3-BDH, AR or diacetyl reductase was similar $(E$-value $<8 \mathrm{e}-112)$ to BudC protein. Moreover, the BudC protein sequence has a very low identity with the BDH found in other Bacillus species. For example, it has only $11.67 \%, 9.98 \%$ and $11.58 \%$ similarity to the BDHs from $B$. subtilis 168 (NP_388505.1), B. cereus YUF-4 (BAB60856.1) and B. amyloliquefaciens DSM 7 (YP_003919213.1), respectively. All these results indicate that unique $\mathrm{BDH}$-encoding gene in B. licheniformis is different from other Bacillus genus.

Although the deletion of budC gene caused a slight decrease (about 5 to $10 \%$ ) in cell growth (Figure 6C), it significantly enhanced the $D$-2,3-butanediol production (Figure 6C) $(30.76 \mathrm{~g} / \mathrm{L})$ compared to both the wild strain in this work and the genetically modified strain with the deletion of the $l d h$ gene $(13.77 \mathrm{~g} / \mathrm{L})$ [6]. Finally, it should be noted that even when budC was deleted from the B. licheniformis WX-02 genome, there was still a small amount of meso-2,3-butanediol found at the end of fermentation period (Figure 6A). This may be due to the existence of other genes encoding minor meso-BDHs, or acetylacetoin reductase catalyzing acetoin to meso-2,3-butanediol [18]. Indeed, low concentration of glucose and high concentration of acetoin, as found in this work (Figure 6A and C), can induce acetylacetoin synthase to transform acetoin to meso-2,3-butanediol through the 2,3-butanediol cycle [18]. 


\section{Conclusions}

In summary, this report revealed the specific function of budC for the transformation between acetoin and 2, 3-butanediol in B. licheniformis. The D-2,3-butanediol production level obtained in this work was the highest among the reported Bacillus genus. The study provides a deep understanding of acetoin and 2,3-butanediol metabolism in B. licheniformis, and a possible way for enhancing the production of pure $D$-2,3-butanediol isomer through genetic modification.

\section{Materials and methods}

\section{Cell strain, plasmids, primers and growth media}

Experiments were performed with the strains and plasmids listed in Table 1. The oligonucleotide primers listed in Table 2 were designed on the basis of B. licheniformis WX-02 genome sequence [GenBank: AHIF00000000] [16]. Luria-Bertani (LB) medium was prepared for culture of E. coli DH5 $\alpha$ and also B. licheniformis [23]. Medium used for culturing $B$. licheniformis was a slight modification of that described in a previous report [24], consisting of (per liter) 120 g glucose, 33 g corn steep liquor, $9.00 \mathrm{~g}\left(\mathrm{NH}_{4}\right)_{2} \mathrm{SO}_{4}, 1.00 \mathrm{~g} \mathrm{~K}_{2} \mathrm{HPO}_{4}, 1.50 \mathrm{~g} \mathrm{MgSO}_{4}$, $0.50 \mathrm{~g} \mathrm{NaCl}, 0.12 \mathrm{~g} \mathrm{ZnCl}_{2}, 1 \mathrm{mg} \mathrm{FeCl}_{3}$, and $1 \mathrm{mg}$ $\mathrm{MnSO}_{4}$. The medium was adjusted to 7.0 before autoclaving at $121^{\circ} \mathrm{C}$ for 15 minutes.

\section{Chemicals and materials for cloning}

Acetoin (98\%) and 2,3-butanediol (98\%) were purchased from Shanghai Jingchun Reagent (China). D-2,3-butanediol ( $>96 \%)$ was purchased from Tokyo Chemical Industry (Tokyo, Japan), meso-2,3-butanediol (99\%) was purchased from Sigma-Aldrich (Sigma, St. Louis, MO, USA). All other chemicals were of analytical grade supplied by
Sinopharm Chemical Reagent (Shanghai, China). T4 DNA ligase and DNA marker were purchased from Takara Bio (Dalian, China). TransStart FastPfu DNA Polymerase was purchased from TransGen Biotech (Beijing, China). Plasmid Miniprep Kit was obtained from Zoman Biotech (Beijing, China). Nucleotide sequences were determined by Beijing Genomics institution (Beijing, China).

\section{Construction of plasmids}

B. licheniformis WX-02 or B. subtilis 168 was cultured in LB medium overnight, and then collected for extraction of genomic DNA based on the method described previously [25]. The extracted genomic DNA was stored at $-20^{\circ} \mathrm{C}$ prior to use. The gene budC was deleted by the doublecrossover homologous recombination method with the primers listed in Table 2. First, two homologous arms (homologous to the $5^{\prime}$ and $3^{\prime}$ coding regions of the budC gene) of approximately $500 \mathrm{bp}$ were amplified by PCR from the genomic DNA of $B$. licheniformis WX-02 by primers of $\Delta b u d \mathrm{C}-\mathrm{A}-\mathrm{F}$ and $\Delta b u d \mathrm{C}-\mathrm{A}-\mathrm{R}, \Delta b u d \mathrm{C}-\mathrm{B}-\mathrm{F}$ and $\Delta b u d \mathrm{C}-\mathrm{B}-\mathrm{R}$, respectively. These two homologous arms were ligated by splicing with overlapping extension $\mathrm{PCR}$ (SOE-PCR) with primers of $\Delta b u d \mathrm{C}-\mathrm{A}-\mathrm{F}$ and $\Delta b u d \mathrm{C}-\mathrm{B}-\mathrm{R}$ [6]. The DNA fragment was subcloned in vector T2(2)-ori joined by $\mathrm{BamH} \mathrm{I}$ and Xba I restriction sites. T2(2)-ori was a previously constructed shuttle plasmid for construction of knock-out vector for B. licheniformis, with a temperature-sensitive replicon from $B$. subtilis to promote single crossover in bacterial cells [26]. The resulting plasmid was further verified by sequencing. A recombinant vector for budC knock-out was designated as T2 $\Delta b u d \mathrm{C}$ (Figure $2 \mathrm{~A}$ ).

The fusion of the $\mathrm{P} 43$ promoter of B. subtilis 168, budC gene of WX-02, and terminator of $a m y \mathrm{~L}$ gene of

Table 1 Bacterial strains and plasmids used in this study

\begin{tabular}{|c|c|c|}
\hline Strains and plasmids & Characteristics $^{\mathrm{a}}$ & Source or reference \\
\hline \multicolumn{3}{|l|}{ E. coli strains } \\
\hline $\mathrm{DH} 5 \mathrm{a}$ & $\begin{array}{l}\mathrm{F}^{-} \text {Ф80d/lacZ } \Delta \mathrm{M} 15, \Delta(\text { lacZYA-argF }) \cup 169 \text {, recA1, endA1, hsdR17 }\left(r_{\mathrm{K}}^{-}, m_{\mathrm{K}}{ }^{+}\right) \text {, } \\
\text { phoA, supE44, } \lambda^{-} \text {, thi-1, gyrA96, relA1 }\end{array}$ & Laboratory stock \\
\hline \multicolumn{3}{|l|}{ B. licheniformis strains } \\
\hline WX-02 & CCTCC M208065, wild type & Laboratory stock [14] \\
\hline WX-02 $\Delta$ budC & budC knock-out mutant of WX-02 & This study \\
\hline WX-02 $\Delta$ budC/pHYbudC & $\begin{array}{l}\text { plasmid-based budC complementation strain of WX-02 } \Delta \text { budC by introduction } \\
\text { of pHYbudC, } \mathrm{TC}^{r}\end{array}$ & This study \\
\hline \multicolumn{3}{|l|}{ Plasmids } \\
\hline T2(2)-ori & E. coli-B. licheniformis shuttle vector, ori ${ }_{p u d}$ /ori $_{t s}$, temperature-sensitive, $\mathrm{Kan}^{r}$ & Laboratory stock \\
\hline T2 $\Delta$ budC & T2(2)-ori derivative containing homologous arms for budC knock-out & This study \\
\hline pHY300PLK & E. coli-B. licheniformis shuttle vector, $\mathrm{Ap}^{\mathrm{r}}\left(E\right.$. coli), $\mathrm{TC}^{\mathrm{r}}$ (E. coli and B. licheniformis) & TaKaRa \\
\hline pHYbudC & $\begin{array}{l}\text { pHY300PLK derivative containing budC, P43 promoter and TamyL (amyL } \\
\text { terminator), } \mathrm{Ap}^{\mathrm{r}}\left(\text { E. coli), } \mathrm{TC}^{\mathrm{r}}(\text { E. coli and B. licheniformis) }\right.\end{array}$ & This study \\
\hline
\end{tabular}

${ }^{\mathrm{a}} \mathrm{TC}^{\mathrm{r}}$, tetracycline resistance; $\mathrm{Ap}^{\mathrm{r}}$, ampicillin resistance; ori $\mathrm{i}_{\mathrm{t},}$, temperature-sensitive Bacillus origin of replication; Kan ${ }^{\mathrm{r}}$, Kanamycin resistance; CCTCC, China Center for Type Culture Collection. 
Table 2 Primers used in this study

\begin{tabular}{|c|c|}
\hline Primer name & asequence $5^{\prime} \rightarrow 3^{\prime}$ \\
\hline$\Delta b u d C-A-F$ & CGCGGATCCAAAGCGCATGTITAAAAC \\
\hline$\Delta b u d C-A-R$ & CCGCCCTCCATATAGAATATAATTTTAAAAATAAACATCTTCTTTCTATAAGTAA \\
\hline$\Delta b u d C-B-F$ & ACCAATTACTTATAGAAAGAAGATGTTTATTTTTAAAATTATATTCTATATGGAG \\
\hline$\Delta b u d C-B-R$ & GCTCTAGACCTCGCACTAGTGTATTTTGAAAC \\
\hline$\Delta b u d C-F$ & CGAACTCCATGAACTGACAGTC \\
\hline$\Delta b u d C-R$ & TTGCTATTTCCTGTTATGACC \\
\hline P43-budC-TamyL-1 & CGCGGATCCTGTCGACGTGCATGCAGG \\
\hline P43-budC-TamyL-2 & CAATTTTTCCAGATACTTTACTCATGTGTACATTCCTCTCTTACCTATA \\
\hline P43-budC-TamyL-3 & TATAGGTAAGAGAGGAATGTACACATGAGTAAAGTATCTGGAAAAATTG \\
\hline P43-budC-TamyL-4 & CGTCCTCTCTGCTCTTCTATCTTTTAATTAAATACCATTCCGCCATC \\
\hline P43-budC-TamyL-5 & GATGGCGGAATGGTATTTAATTAAAAGATAGAAGAGCAGAGAGGACG \\
\hline P43-budC-TamyL-6 & CCGGAATTCGATCACCCGCGATACCGTC \\
\hline$\Delta b u d C$ A signal crossover- $F$ & CTTCACATGGACGATCCTAAT \\
\hline$\Delta$ budC A single crossover- $R$ & TGTTCCTCCGTAAACCGCTAAG \\
\hline$\Delta$ budC B single crossover- $F$ & CAACCACCCCTATTGAAAGCAT \\
\hline$\Delta$ budC B single crossover- $R$ & GATACCTGTCCGCCTTTCTCC \\
\hline
\end{tabular}

${ }^{a}$ Restriction sites highlighted in bold. Italics stands for the overlap region for splicing by overlapping extension PCR (SOE-PCR).

B. licheniformis WX-02 were achieved by SOE-PCR with primers of P43-budC-TamyL-1 to 6 (Table 2) and templates of genomic DNA from B. licheniformis WX-02 or B. subtilis 168. Then the DNA fragment amplified by SOE-PCR was cloned into the plasmid of pHY300PLK joined by the $B a m \mathrm{H}$ I and EcoR I restriction sites. The resulting plasmid was verified by sequencing. A recombinant vector for expression of budC in B. licheniformis WX-02 was designated as pHYbudC (Figure 2B).

\section{Construction of the budC knock-out strain of WX-02}

Competent cells of E. coli DH5 $\alpha$ and B. licheniformis WX-02 were prepared for transformation of constructed plasmids as described previously [23,27]. E. coli $\mathrm{DH} 5 \alpha$ was transferred with T2 $\Delta b u d C$ plasmid and cultured in LB medium with kanamycin $(20 \mu \mathrm{g} / \mathrm{mL})$. The plasmid $\mathrm{T} 2 \Delta b u d \mathrm{C}$ isolated from the recombinant E. coli $\mathrm{DH} 5 \alpha$ was used for transforming into B. licheniformis WX-02.

B. licheniformis WX-02 was electrotransformed with the recombinant $\mathrm{T} 2 \Delta b u d C$ plasmid according to the method described previously [27]; the transformants were selected by kanamycin resistance $(20 \mu \mathrm{g} / \mathrm{mL})$ followed with verification by PCR using the primers $\Delta b u d \mathrm{C}-\mathrm{A}-\mathrm{F}$ and $\Delta b u d \mathrm{C}$ B-R (Table 2). The selected positive transformant was cultured in LB medium containing kanamycin $(20 \mu \mathrm{g} / \mathrm{mL})$ at $45^{\circ} \mathrm{C}$ for $8 \mathrm{~h}$, and the temperature-sensitive replicon of the T2 $\Delta b u d C$ plasmid did not work at this temperature. Therefore, the high growth temperature promoted the first crossover in the cells. The mutants with kanamycin resistance were selected, and further verified by $\mathrm{PCR}$ with primers of $\Delta b u d \mathrm{C}$ A single crossover-F and $\Delta b u d \mathrm{C}$ A single crossover- $\mathrm{R}$ for crossover upstream, or $\Delta b u d \mathrm{C}$ B single crossover-F and $\Delta b u d \mathrm{C} B$ single crossover- $\mathrm{R}$ for crossover downstream. Then the selected colonies with single crossover were picked up and cultured in LB medium at $37^{\circ} \mathrm{C}$ for 8 hours, this process was repeated six times. After serial transfer without antibiotics, cells were plated on LB agar plates, and then replicated in kanamycin plates for selection of kanamycin-sensitive colonies. The budC knock-out strains that had looped out the kanamycin-resistant gene by the second crossover were selected. The mutant WX-02 $\Delta b u d \mathrm{C}$ was confirmed by PCR with primers of $\Delta b u d C$-F and $\Delta b u d C-\mathrm{R}$ (Table 2) and nucleotide sequencing.

\section{Construction of the complementary strain of WX-02 $\Delta$ budC}

The complementation of $B$. licheniformis WX-02 $\Delta b u d C$ was conducted with a budC expression plasmid. The $B$. licheniformis WX-02 $\Delta b u d \mathrm{C}$ was electrotransformed with pHYbudC DNA according to the method described previously [27], and the transformants were first selected by LB agar plates with $20 \mu \mathrm{g} / \mathrm{mL}$ tetracycline [27], followed with verification by PCR with primers of P43-budC-TamyL-1 and P43-budC-TamyL-6 (Table 2). The recombinant strain was designated as WX-02 $\Delta b u d \mathrm{C} / \mathrm{pHY} b u d \mathrm{C}$.

\section{Detection of BDH and AR activities in cells}

The wild strain WX-02, mutant strain WX-02 $\Delta b u d C$, and complementary strain WX-02 $\Delta b u d \mathrm{C} / \mathrm{pHY} b u d \mathrm{C}$ were cultured for 12, 24 and $36 \mathrm{~h}$. The cell extracts from these three cultures were prepared for determining the 2,3-BDH 
and AR activity based on the previous methods [11]. The reaction system contains $4 \mathrm{mmol} / \mathrm{L} \mathrm{NAD}^{+}$and $100 \mathrm{mmol} /$ $\mathrm{L}$ 2,3-butanediol for the BDH assay or $0.2 \mathrm{mmol} / \mathrm{L} \mathrm{NADH}$ and $50 \mathrm{mmol} / \mathrm{L}$ acetoin for the AR assay [11]. The cell extracts and reaction system were preheated at $37^{\circ} \mathrm{C}$, the 200- $\mu \mathrm{L}$ reaction system was then added to a 96-well UVstar microplate (Greiner Bio-One, Germany) followed with addition of $5 \mu \mathrm{L}$ cell extracts. The microplate was immediately put into a microplate reader (BioTek, USA) and reacted at $37^{\circ} \mathrm{C}$ for 5 minutes. Absorbance at $340 \mathrm{~nm}$ was measured initially and the end of the reaction. Under these conditions, one unit of $\mathrm{BDH}$ or AR activity was defined as $1 \mu \mathrm{mol}$ of NADH produced or consumed by $1 \mathrm{mg}$ of protein per minute. The protein concentration of cell extracts was determined by the Coomassie brilliant blue method [28].

\section{Analysis}

Cell density was determined by the optical absorbance at $600 \mathrm{~nm}\left(\mathrm{OD}_{600}\right)$. The concentration of residual glucose was measured by a biosensor equipped with a glucoseoxidase electrode (SBA-40C, China). Single colonies of the wild strain of WX-02, mutant strain of WX-02 $\Delta b u d \mathrm{C}$, and complementary strain of WX-02 $\Delta b u d \mathrm{C} / \mathrm{pHY} b u d \mathrm{C}$ on the LB plate were transferred into 250 -mL flasks containing $50 \mathrm{~mL} \mathrm{LB}$ medium and incubated at $37^{\circ} \mathrm{C}$ for $11 \mathrm{~h}$ in an orbital shaker at $180 \mathrm{rpm}$ until the $\mathrm{OD}_{600}$ of the culture reached approximately 4.2. The cells were then sub-cultured for $48 \mathrm{~h}$ in the same conditions. The samples were collected periodically to determine the time course of cell density, residual glucose, and product (acetoin and 2,3-butanediol) concentrations using previously described methods [29].

Acetoin, D-2,3-butanediol, and meso-2,3-butanediol were extracted by ethyl acetate and then quantified using Trace GC Ultra Gas Chromatograph (Thermo, USA) equipped with a flame ionization detector and TR-WAX capillary column (30 $\mathrm{m} \times 0.32 \mathrm{~mm}$ ID, $0.25 \mu \mathrm{m}$ film). Nitrogen was used as the carrier gas with a flow rate of $1.0 \mathrm{~mL} /$ minute; the injected volume was $1 \mu \mathrm{L}$ with a splitless injection mode. The injector temperature and the detector temperature were $215^{\circ} \mathrm{C}$ and $245^{\circ} \mathrm{C}$, respectively. The column was maintained at $50^{\circ} \mathrm{C}$ for 1.5 minutes, increased at a rate of $10^{\circ} \mathrm{C} /$ minute to $110^{\circ} \mathrm{C}$ for 0.5 minutes, $5^{\circ} \mathrm{C} /$ minute to $150^{\circ} \mathrm{C}$ for 0.5 minutes, and $20^{\circ} \mathrm{C} /$ minute to $220^{\circ} \mathrm{C}$ for $1 \mathrm{mi}-$ nute. The concentration of acetoin and 2,3-butanediol was quantified using the internal standard (butanol).

\footnotetext{
Abbreviations

Amy: amylase; AR: acetoin reductase; $\mathrm{BDH}$ : 2,3-butanediol dehydrogenase; BDL: butanediol; bp: base pairs; GC: gas chromatograph; Ldh: lactate dehydrogenase; NADH: nicotinamide adenine dinucleotide; OD: optical density; SOE-PCR: splicing with overlapping extension PCR; Y-PGA: $\gamma$-poly-glutamic acid.
}

\section{Competing interests}

The authors declare that they have no competing interests.

\section{Authors' contributions}

$\mathrm{GQ}$ conceived of the study, performed the data analysis, and coordinated the manuscript draft and revision. YK and LL executed the experimental work and data analysis. AX and SZ executed the experimental work. ZW helped to revise and proofread the manuscript. DX helped with data analysis. SC conceived the study, and coordinated the manuscript draft and revision. All authors read and approved the final manuscript.

\section{Acknowledgements}

This work was supported by the National Natural Science Foundation of China (Grant No.31170046 and J1103510). This work was also supported by rural areas of the national science and technology plan in the 12th five-year plan of China (No.2013AA102801-52).

\section{Author details}

${ }^{1}$ State Key Laboratory of Agricultural Microbiology, College of Life Science and Technology, Huazhong Agricultural University, Wuhan 430070, China. ${ }^{2}$ Department of Food Science and Human Nutrition, lowa State University, Ames, IA 50011, USA. ${ }^{3}$ College of Food Science and Technology, Huazhong Agricultural University, Wuhan 430070, China.

Received: 30 October 2013 Accepted: 14 January 2014

Published: 29 January 2014

\section{References}

1. Gao J, Yang HH, Feng XH, Li S, Xu H: A 2,3-butanediol dehydrogenase from Paenibacillus polymyxa ZJ-9 for mainly producing R, R-2,3-butanediol: purification, characterization and cloning. J Basic Microbio/ 2012, 52:1-9.

2. Ji XJ, Huang H, Ouyang PK: Microbial 2,3-butanediol production: a state-of-the-art review. Biotechnol Adv 2011, 29:351-364.

3. Yu B, Sun J, Bommareddy RR, Song L, Zeng AP: Novel (2R,3R)-2,3butanediol dehydrogenase from potential industrial strain Paenibacillus polymyxa ATCC 12321. Appl Environ Microbiol 2011, 77:4230-4233.

4. Xiao Z, Lv C, Gao C, Qin J, Ma C, Liu Z, Liu P, Li L, Xu P: A novel whole-cell biocatalyst with $\mathrm{NAD}^{+}$regeneration for production of chiral chemicals. PLoS One 2010, 5:e8860.

5. Liu Z, Qin J, Gao C, Hua D, Ma C, Lia L, Wanga Y, Xu P: Production of $(2 S, 3 S)-2,3$ - butanediol and (3S)-acetoin from glucose using resting cells of Klebsiella pneumonia and Bacillus subtilis. Bioresour Technol 2011, 102:10741-10744.

6. Wang QZ, Chen T, Zhao X, Chamu J: Metabolic engineering of thermophilic Bacillus licheniformisfor chiral pure D-2,3-butanediol production. Biotechnol Bioeng 2012, 109:1610-1621.

7. Siemerink MA, Kuit W, Contreras AML, Eggink G, van der Oost J, Kengen SW: D - 2, 3 - butanediol production due to heterologous expression of an acetoin reductase in Clostridium acetobutylicum. App/ Environ Microbiol 2011, 77:2582-2588.

8. Li J, Wang W, Ma Y, Zeng AP: Medium optimization and proteome analysis of $(R, R)$ - 2,3 - butanediol production by Paenibacillus polymyxa ATCC 12321. Appl Microbiol Biot 2013, 97:585-597.

9. Jurchescu IM, Hamann J, Zhou X, Ortmann T, Kuenz A, Prüße U, Lang S: Enhanced 2,3-butanediol production in fed-batch cultures of free and immobilized Bacillus licheniformis DSM 8785. Appl Microbiol Biot 2013, 97:6715-6723.

10. Li L, Zhang L, Li K, Wang Y, Gao C, Han B, Ma C, Xu P: A newly isolated Bacillus licheniformis strain thermophilically produces 2,3-butanediol, a platform and fuel biochemical. Biotechnol Biofuels 2013, 6:123.

11. Nicholson WL: The Bacillus subtilis ydjL (bdhA) gene encodes acetoin reductase/2,3 - butanediol dehydrogenase. Appl Environ Microbiol 2008, 74:6832-6838.

12. Nielsen DR, Yoon SH, Yuan CJ, Prather KLJ: Metabolic engineering of acetoin and meso-2,3-butanediol biosynthesis in E. coli. Biotechnol J 2010, 5:274-284.

13. Li L, Wang Y, Zhang L, Ma C, Wang A, Tao F, Xu P: Biocatalytic production of $(2 S, 3 S)-2,3$-butanediol from diacetyl using whole cells of engineered Escherichia coli. Bioresour Technol 2012, 115:111-116.

14. Wei XT, Ji ZX, Chen SW: Isolation of halotolerant Bacillus licheniformis WX02 and regulatory effects of sodium chloride on yield and molecular 
sizes of poly-ү-glutamic acid. Appl Biochem Biotechnol 2010, 160:1332-1340.

15. Renna MC, Najimudin N, Winik $L R$, Zahler $S A$ : Regulation of the Bacillus subtilis als $\mathrm{S}$, alsD, and alsR genes involved in post-exponential-phase production of acetoin. J Bacteriol 1993, 175:3863-3875.

16. Yangtse W, Zhou Y, Lei Y, Qiu Y, Wei X, Ji Z, Qi G, Yong Y, Chen L, Chen S: Genome Sequence of Bacillus licheniformis WX-02. J Bacteriol 2012, 194:3561-3562.

17. Giovannini PP, Mantovani M, Medici A, Pedrini P: Production of 2,3butanediol by Bacillus stearothermophilus: fermentation and metabolic pathway. Chem Eng 2008, 14:281-286.

18. Giovannini PP, Mantovani M, Grandini A, Medici A, Pedrini P: New acetoin reductases from Bacillus stearothermophilus: meso- and 2R, 3Rbutanediol as fermentation products. J Mol Catal B Enzym 2011, 69:15-20.

19. Zhang XZ, Cui ZL, Hong Q, Li SP: High-level expression and secretion of methyl parathion hydrolase in Bacillus subtilis WB800. Appl Environ Microbiol 2005, 71:4101-4103.

20. Rao B, Zhang LY, Sun J, Su G, Wei D, Chu J, Zhu J, Shen Y: Characterization and regulation of the 2,3-butanediol pathway in Serratia marcescens. Appl Microbiol Biot 2012, 93:2147-2159.

21. Ali NO, Bignon J, Rapoport G, Debarbouille M: Regulation of the acetoin catabolic pathway is controlled by sigma $\mathrm{L}$ in Bacillus subtilis. J Bacteriol 2001, 183:2497-2504.

22. Thanh TN, Jürgen B, Bauch M, Liebeke M, Lalk M, Ehrenreich A, Evers S, Maurer K, Antelmann H, Ernst F, Homuth G, Hecker M, Schweder T: Regulation of acetoin and 2, 3-butanediol utilization in Bacillus licheniformis. Appl Microbiol Biotechnol 2010, 87:2227-2235.

23. Sambrook J, Russell DW: Molecular Cloning: A Laboratory Manual. The 3rd ed., Cold Spring Horbor laboratory [Z]. NY: Cold Spring Harbor; 2001.

24. Liu Y, Zhang S, Yong YC, Ji Z, Ma X, Xu Z, Chen S: Efficient production of acetoin by the newly isolated Bacillus licheniformis strain MEL09. Process Biochem 2011, 46:390-394.

25. Sohail M: A simple and rapid method for preparing genomic DNA from gram-positive bacteria. Mol Biotechnol 1998, 10:191-193.

26. Guerout-Fleury AM, Shazand K, Frandsen N, Stragier P: Antibiotic-resistance cassettes for Bacillus subtilis. Gene 1995, 167:335-336.

27. Xue G, Johnson JS, Dalrymple BP: High osmolarity improves the electrotransformation efficiency of the gram-positive bacteria Bacillus subtilis and Bacillus licheniformis. J Microbiol Meth 1999, 34:183-191.

28. Bradford MM: A rapid and sensitive method for the quantitation of microgram quantities of protein utilizing the principle of protein-dye binding. Anal Biochem 1976, 72:248-254.

29. Gao J, Xu H, Li QJ, Feng XH, Li S: Optimization of medium for one-step fermentation of inulin extract from Jerusalem artichoke tubers using Paenibacillus polymyxa ZJ-9 to produce R, R-2,3-butanediol. Bioresour Technol 2010, 101:7076-7082.

doi:10.1186/1754-6834-7-16

Cite this article as: Qi et al:: Deletion of meso-2,3-butanediol dehydrogenase gene budC for enhanced $D$-2,3-butanediol production in Bacillus licheniformis. Biotechnology for Biofuels 2014 7:16.

\section{Submit your next manuscript to BioMed Central and take full advantage of:}

- Convenient online submission

- Thorough peer review

- No space constraints or color figure charges

- Immediate publication on acceptance

- Inclusion in PubMed, CAS, Scopus and Google Scholar

- Research which is freely available for redistribution

Submit your manuscript at www.biomedcentral.com/submit
Biomed Central 